Check for updates

Cite this: RSC Adv., 2017, 7, 36923

Received 5th June 2017

Accepted 19th July 2017

DOI: $10.1039 / \mathrm{c} 7 \mathrm{ra06206k}$

rsc.li/rsc-advances

\section{Facile synthesis of Au/Pd nano-dogbones and their plasmon-enhanced visible-to-NIR light photocatalytic performance}

\author{
Yueyue Yin, ${ }^{\text {ab }}$ Yong Yang, (D) *a Liangzhu Zhang, ${ }^{\mathrm{cd}}$ Yongsheng Li, (D) ${ }^{\mathrm{b}}$ Zhiyuan Li, \\ Weiwei Lei, (D) ${ }^{d}$ Yunfeng $\mathrm{Ma}^{\mathrm{a}}$ and Zhengren Huang ${ }^{\mathrm{a}}$
}

\begin{abstract}
Here, we report a facile synthesis of Pd/Au nano-dogbones (NDBs) by loading massive Pd nanoparticles on the two ends of $\mathrm{Au}$ nanorods (NRs) via a seed-mediated method. The as-prepared samples were investigated by using TEM, elemental analysis, UV-vis and FDTD simulation. The photocatalytic activity of $\mathrm{Pd} / \mathrm{Au}$ NDBs was assessed by dehydrogenation of formic acid under visible-to-NIR light irradiation. The $\mathrm{Pd} / \mathrm{Au}$ NDBs shows much higher enhanced reaction activity compared with samples with core-shell structure due to the increased electron and hole pair separation and total catalytic sites. More importantly, the introduction of Au NRs broadens the photoresponse range and improves the utilization of sunlight efficiently. This finding provides a rational approach to improve photocatalytic performance by forming dogbone structures via integrating plasmonic metals and conventional catalysts.
\end{abstract}

\section{Introduction}

The demanding energy crisis drives scientists to look for green and abundant fuels. $\mathrm{H}_{2}$ generated directly via photocatalysis has attracted significant notice, in consideration of solar energy sustainable supply and clean utilization. ${ }^{1}$ However, conventional catalysts are restricted in practical application because of their lower catalytic efficiency under visible light irradiation. ${ }^{2,3}$ Therefore, developing an efficient and stable visible-light response catalyst is undoubtedly meaningful. Plasmoic metal nanostructures greatly promote the visible light to NIR absorption due to surface plasmon resonances (SPR). More importantly, the separation of hot electrons and holes in plasmonic metals coupled with conventional catalysts has a synergy effect, leading to enhanced catalytic activity. Therefore, rational combining plasmonic metal with traditional catalytic particles to form a heterostructure holds promise in photocatalysis..$^{4-6}$

\footnotetext{
${ }^{a}$ State Key Laboratory of High Performance Ceramics and Superfine Microstructure, Shanghai Institute of Ceramics, Chinese Academy of Sciences, 1295 Dingxi Road, Shanghai 200050, China. E-mail: yangyong@mail.sic.ac.cn

${ }^{b}$ Lab of Low-Dimensional Materials Chemistry, Key Laboratory for Ultrafine Materials of Ministry of Education, School of Materials Science and Engineering, East China University of Science and Technology, Shanghai 200237, China

'Key Laboratory of Inorganic Functional Materials and Devices, Shanghai Institute of Ceramics, Chinese Academy of Sciences, 215 Chengbei Road, Jiading, Shanghai 201800, China

${ }^{d}$ Institute for Frontier Materials, Deakin University, Waurn Ponds Campus, Victoria 3216, Australia

${ }^{e}$ Laboratory of Optical Physics, Institute of Physics, Chinese Academy of Sciences, P. O. Box 603, Beijing 100190, China
}

Au NRs have triggered scientist interests for the ability to harvest a wide range of visible light and even NIR light due to tunable SPR, which contributes to improve solar energy conversion efficiency. For example, Au NRs integrated with $\mathrm{TiO}_{2}$ or Pt showed enhanced catalytic activity in water splitting ${ }^{7,8}$ However, little effort was allocated to integrating Au NRs with catalytic catalyst for $\mathrm{H}_{2}$ production from formic acid under visible light irradiation. Generally, $\mathrm{H}_{2}$ is generated via a catalytically dehydrogenation pathway ( $\mathrm{HCOOH} \mathrm{(l)} \rightarrow \mathrm{H}_{2}(\mathrm{~g})+\mathrm{CO}_{2}$ (g), $\Delta G_{298}=-35.0 \mathrm{~kJ} \mathrm{~mol}^{-1}$ ), while, carbon monoxide (CO), a disastrous poison to catalysts, can also be generated through a dehydration route $\left(\mathrm{HCOOH}(\mathrm{l}) \rightarrow \mathrm{H}_{2} \mathrm{O}(\mathrm{l})+\mathrm{CO}(\mathrm{g}), \Delta G_{298}=\right.$ $\left.-14.9 \mathrm{~kJ} \mathrm{~mol}^{-1}\right){ }^{9-11}$ For the purpose of improving hydrogen production efficiency, it is obviously desirable to develop novel catalysts for directly $\mathrm{H}_{2}$ conversion. Inspiring, much progress has been made on heterogeneous catalysis for selective dehydrogenation of formic acid. ${ }^{12-17}$ Among different catalytic metals, Pd-based catalysts are most promising for practical hydrogen production from formic acid. ${ }^{18-22}$ However, the absence of strong SPR absorption over Pd inhibited light energy utilization. ${ }^{23-25}$ Recently, Pd single layer tipped Au NRs demonstrated efficient plasmon-enhanced catalytic formic acid dehydrogenation even when below room temperature $\left(5^{\circ} \mathrm{C}\right) .{ }^{26}$ However, there are not enough electron-hole separation channels in Pd single-crystal covered structure, which would hinder the further improvement on catalytic properties. If more Pd poly-crystal nano particles (NPs) covered on two ends of Au NRs, more $\mathrm{Pd}-\mathrm{Au}$ catalytic active sites and more electron-hole separation channels will be generated due to enlarged surface area of more Pd NPs. Therefore, it is one promising route to improve the photocatalytic activity by smaller Pd polycrystalline 


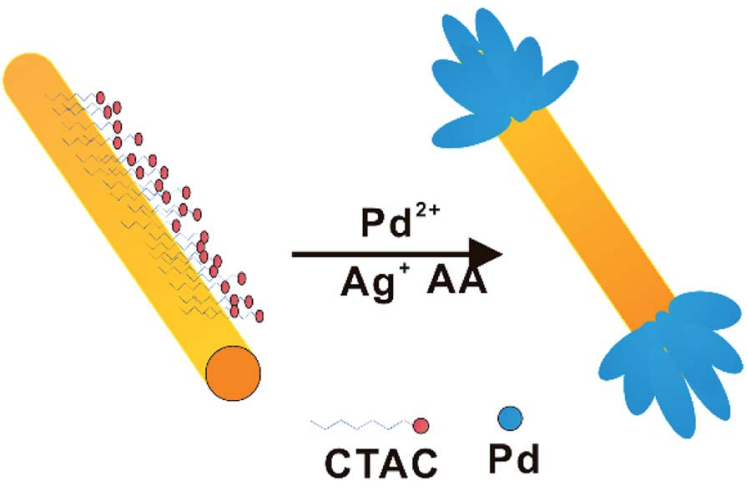

Scheme 1 Synthetic procedure for Au/Pd NDBs.

NPs covered Au NRs. However, to synthesize such a well-defined spatial separation structure by all wet-chemistry routes is challenging.

Herein, we developed an efficient method for synthesis of $\mathrm{Au} / \mathrm{Pd}$ NDBs via the reduction of $\mathrm{Pd}^{2+}$ by L-ascorbic acid (AA) in the presence of CTAC-capped $\mathrm{Au} \mathrm{NRs}$ and $\mathrm{Ag}^{+}$. As schematic illustration of the process is shown in Scheme 1. By controlling the CTAC bilayer confines the Au NR with only two ends accessible to Pd species. Ag was first deposited on nanorods, then was consumed by the $\mathrm{Pd}^{2+}$ ions in the solution via galvanic replacement reaction, leading to the formation of dogbones structure. ${ }^{27,28}$ The Au/Pd NDBs have a spatial separation structure, and Pd act as acceptor for hot electrons from Au NRs. This structure satisfies electron refilling requirement and exhibits plasmon-enhanced hydrogen production from formic decomposition under visible and NIR light irradiation.

\section{Experimental}

\subsection{Chemicals}

All chemicals were purchased from commercial corporations. CTAC (96\% powder), sodium oleate (NaOL, >97.0\%) and sodium borohydride $\left(\mathrm{NaBH}_{4}, 99 \%\right)$ were purchased from Aladdin. Hydrogen tetrachloraurate trihydrate $\left(\mathrm{HAuCl}_{4} \cdot 3 \mathrm{H}_{2} \mathrm{O}\right)$, silver nitrate $\left(\mathrm{AgNO}_{3}, 99.99 \%\right), \mathrm{CTAB}(>98.0 \%)$, L-ascorbic acid (BioUltra, $>99.5 \%)$ and palladium chloride $\left(\mathrm{PdCl}_{2},>99.9 \%\right)$ were purchased from Sigma-Aldrich. Ultrapure water $(10 \mathrm{M} \Omega$ resistivity) was used for all experiments. $10 \mathrm{mM} \mathrm{H}_{2} \mathrm{PdCl}_{4}$ solution was obtained via mixing $44.5 \mathrm{mg}$ of $\mathrm{PdCl}_{2}$ in $25 \mathrm{~mL}$ of $10 \mathrm{mM}$ $\mathrm{HCl}$. All glass ware were treated by freshly prepared aqua regia $\left(\mathrm{HCl}: \mathrm{HNO}_{3}\right.$ in a $3: 1$ ratio by volume) and washed thoroughly with great amount water to remove chemical remains.

\subsection{Preparation of Au NRs}

$\mathrm{Au}$ NRs were prepared by a modified seed-mediated growth method. ${ }^{29,30}$ Generally, the seed solution was obtained by reduction of $\mathrm{HAuCl}_{4} \cdot 3 \mathrm{H}_{2} \mathrm{O}(0.5 \mathrm{mM}, 5 \mathrm{~mL})$ in CTAB $(0.2 \mathrm{M}, 5 \mathrm{~mL})$ solution with ice-cold $\mathrm{NaBH}_{4}(6 \mathrm{mM}, 1 \mathrm{~mL})$. The seed solution was aged at room temperature for $30 \mathrm{~min}$ before use. The obtained seed solution $(0.4 \mathrm{~mL})$ was added into growth solution prepared by the sequential addition of $\mathrm{AgNO}_{3}(24 \mathrm{~mL}, 4 \mathrm{mM})$,
$\mathrm{HAuCl}_{4} \cdot 3 \mathrm{H}_{2} \mathrm{O}(250 \mathrm{~mL}, 1 \mathrm{~mL}), \mathrm{HCl}(4.8 \mathrm{~mL}, 37 \mathrm{wt} \%)$ and $\mathrm{AA}(1.25$ $\mathrm{mL}, 64 \mathrm{mM}$ ) into $250 \mathrm{~mL}$ CTAB-NaOL solution (7.0 g CTAB and $1.234 \mathrm{~g}$ NaOL dissolved in $250 \mathrm{~mL}$ purified water). The resulting $\mathrm{Au}$ NRs were divided into two parts, Au-NRs-1 were redispersed in $25 \mathrm{~mL}$ of $0.1 \mathrm{M} \mathrm{CTAB}$, the as-prepared Au NRs were labeled as AuNRs-1. The other redispersed in 0.1 M CTAC were labeled as AuNRs-2.

\subsection{Preparation of $\mathrm{Au} / \mathrm{Pd} \mathrm{NDBs}$}

The Au-NRs-2 (2 mL) was injected to purified water $(18 \mathrm{~mL})$. $\mathrm{H}_{2} \mathrm{PdCl}_{4}(3 \mathrm{~mL}, 0.01 \mathrm{M}), \mathrm{AgNO}_{3}(4 \mathrm{~mL}, 1 \mathrm{mM})$ and various volumes AA (0.05 $\mathrm{mM})$ were added sequentially in mixture. The solution were left undisturbed in a water bath at $30{ }^{\circ} \mathrm{C}$ for $12 \mathrm{~h}$. The obtained nanostructures were centrifuged twice and redispersed in purified water $(5 \mathrm{~mL})$.

\subsection{Preparation of Au@Pd core-shell nanocuboids}

The Au-NRs-1 (2 mL) was injected to purified water $(18 \mathrm{~mL})$. $\mathrm{H}_{2} \mathrm{PdCl}_{4}(3 \mathrm{~mL}, 0.01 \mathrm{M})$, and $\mathrm{AA}(300 \mu \mathrm{L}, 0.05 \mathrm{mM})$ were added sequentially in mixture. The solution were left undisturbed in a water bath at $30^{\circ} \mathrm{C}$ for $12 \mathrm{~h}$. The obtained nanostructures were centrifuged twice and redispersed in purified water $(5 \mathrm{~mL})$.

\subsection{Preparation of Au@Pd core-shell nanostructure with irregular shapes}

The Au-NRs-2 $(2 \mathrm{~mL})$ was injected to purified water $(18 \mathrm{~mL})$. $\mathrm{H}_{2} \mathrm{PdCl}_{4}(3 \mathrm{~mL}, 0.01 \mathrm{M})$, and $\mathrm{AA}(300 \mu \mathrm{L}, 0.05 \mathrm{mM})$ were added sequentially in mixture. The solution were left undisturbed in a water bath at $30^{\circ} \mathrm{C}$ for $12 \mathrm{~h}$. The obtained nanostructures were centrifuged twice and redispersed in purified water $(5 \mathrm{~mL})$.

\subsection{Preparation of Au@Pd core-shell nanorods with serrated shapes}

The Au-NRs-1 (2 mL) was injected to purified water $(18 \mathrm{~mL})$. $\mathrm{H}_{2} \mathrm{PdCl}_{4}(3 \mathrm{~mL}, 0.01 \mathrm{M}), \mathrm{AgNO}_{3}(4 \mathrm{~mL}, 1 \mathrm{mM})$ and $\mathrm{AA}(300 \mu \mathrm{L}$, $0.05 \mathrm{mM}$ ) were added sequentially in mixture. The solution were left undisturbed in a water bath at $30{ }^{\circ} \mathrm{C}$ for $12 \mathrm{~h}$. The obtained nanostructures were centrifuged twice and redispersed in purified water $(5 \mathrm{~mL})$.

\subsection{Characterization of materials}

Transmission electron microscopy (TEM) images, Scanning TEM (STEM) images and energy dispersive spectroscopy (EDS) mapping images were obtained on a JEOL JEM-2100F electron microscope operating at $200 \mathrm{KV}$. Absorption spectra was taken using quartz cuvettes of $1 \mathrm{~cm}$ path length on a PerkinElmer Lambda 950 instrument.

\subsection{FDTD calculation}

The computational simulations were performed by using finitedifference-time-domain (FDTD) method with perfectly matched layers (PML) boundary conditions. The optical constants of $\mathrm{Au}$ were employed the results of tabulated values for bulk gold. ${ }^{31}$ The size of the NRs was adapted the average values. Particularly, the Au NR was modelled as a cylinder, with a length of $128 \mathrm{~nm}$ 
and width of $27 \mathrm{~nm}$. For the model of Au/Pd NDBs, cylinder Pd nanorods with a length of $15 \mathrm{~nm}$ and width of $7 \mathrm{~nm}$ accumulated at two ends of the Au NR. For NRs dispersed in purified water, the refractive index of the medium was set to be 1.33 .

\subsection{Hydrogen production from formic acid}

The as-prepared catalysts were centrifuged and washed several times. The treated catalyst was added to $100 \mathrm{~mL}$ of formic acid ( $1 \mathrm{M}$ ) and poured into the reaction glassware. For visible-NIR light hydrogen evolution, the reaction mixtures were irradiated for $4 \mathrm{~h}$ with visible-NIR light (PerfectLight, PLS-SXE300, $300 \mathrm{~W}$ ) under magnetic stirring (300 rpm). UV light was removed by a $420 \mathrm{~nm}$ cutoff filter. A water bath was employed to control the reaction temperature at $15{ }^{\circ} \mathrm{C}$. The amount of $\mathrm{H}_{2}$ in the gas phase was measured by Aosong Instrument GC-7890H gas chromatograph equipped with a MS-5A column and a thermal conductivity detector (TCD).

\section{Results and discussion}

Fig. 1a and b show TEM and HRTEM images of the highly monodispersed single-crystalline Au NRs oriented growth along (200) plane by seed-mediated method. Fig. $1 \mathrm{c}$ and d show the prepared $\mathrm{Au}$ NRs presents good uniformity with lengths of $128 \mathrm{~nm}$ and width of $27 \mathrm{~nm}$ (aspect ratio of 4.80). Fig. 1e shows the Au NRs have a wide range of light absorption from $400 \mathrm{~nm}$ to $1200 \mathrm{~nm}$ with LSPR peak centred at about $998 \mathrm{~nm}$, which indicates the $\mathrm{Au}$ NRs are capable harvest the light from UV to visible-NIR.

Fig. 2a-c show TEM images of Au/Pd NDBs obtained in the presence of CTAC-capped $\mathrm{Au} \mathrm{NRs}$ and $\mathrm{Ag}^{+}$. The products are composed of uniform NDBs structure with two ends of Au NRs coated with Pd nano-ball. HRTEM (Fig. 2d) image shows lots of single crystalline Pd nanoparticles with orientated growth along the (200) plane.

The ball size of $\mathrm{Au} / \mathrm{Pd}$ NDBs could be well-controlled by simply modifying the molar ratios of reduction agent AA and Pd atoms

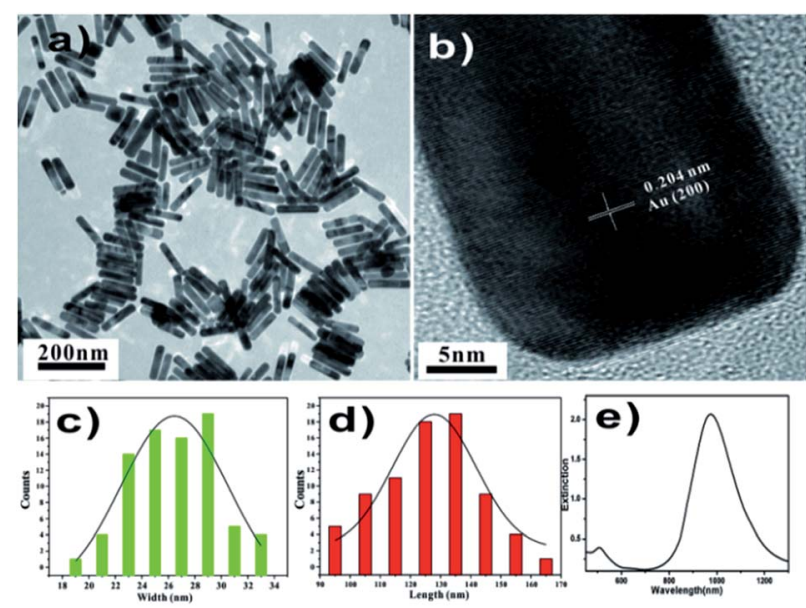

Fig. 1 (a) TEM images of Au NRs, (b) HRTEM of Au NRs, (c and d) size distributions (length and width) of gold NRs, (e) UV-vis-NIR absorption spectra of the Au NRs.
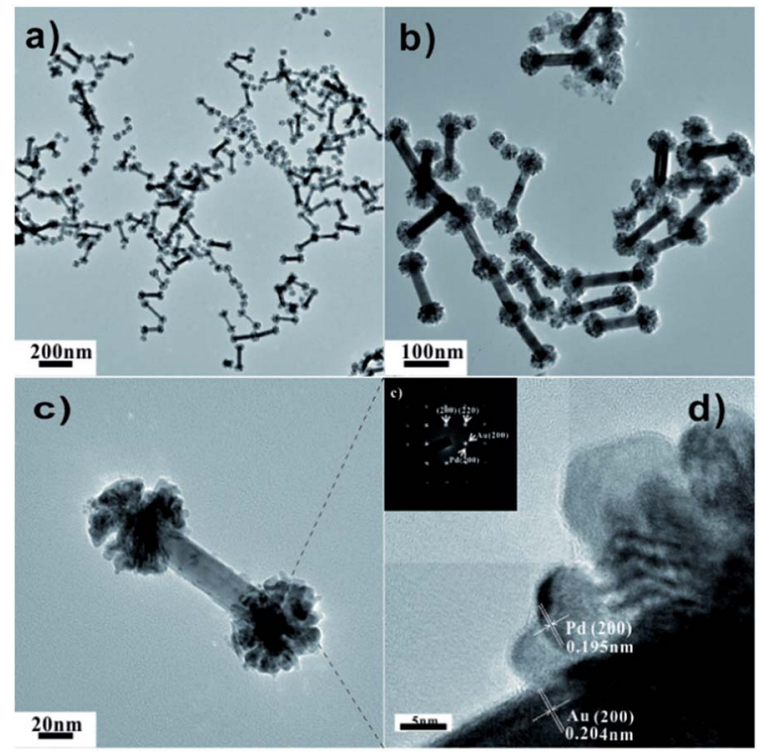

Fig. 2 ( $a$ and b) TEM images of Au/Pd NDs, (c) TEM images of single Au/Pd NDs, (d) HRTEM images of NDs.

source $\mathrm{H}_{2} \mathrm{PdCl}_{4}$. As can be seen in Fig. 3a-d, the ball semidiameter progressively increased form $13 \mathrm{~nm}$ to $44 \mathrm{~nm}$, with molar ratio of AA : $\mathrm{H}_{2} \mathrm{PdCl}_{4}$ (the total amount of $\mathrm{H}_{2} \mathrm{PdCl}_{4}$ was fixed) increasing from 0.1 to 1 . The dissociated nanoshperes are also found. Fig. 4 shows the small variation of LSPR peak of four samples may result in the influence of the ball size on the aspect ratio.

To further identify the element distribution Au/Pd NDBs, STEM and EDS analysis on Au/Pd NDBs were employed. EDS elemental maps of Au/Pd NDBs (Fig. 5) clearly show Pd atoms selectively locate at the ends of Au NRs while no Pd atoms are found in the side of Au NRs, which confirmed the dogbones nanostructure. The Ag mapping present the similar results, shows the ball of the nano-dogbones is PdAg alloy structure. As

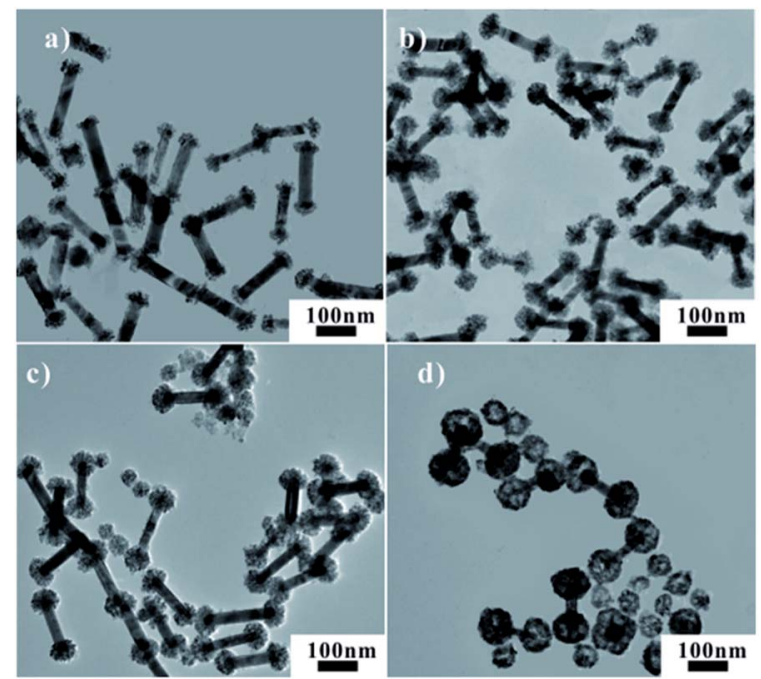

Fig. 3 TEM images of $\mathrm{Au} / \mathrm{Pd} \mathrm{NDBs}$ with various $\mathrm{AA}: \mathrm{H}_{2} \mathrm{PdCl}_{4}$ ratio: (a) 0.1 , (b) 0.25 , (c) 0.5 , (d) 1. 


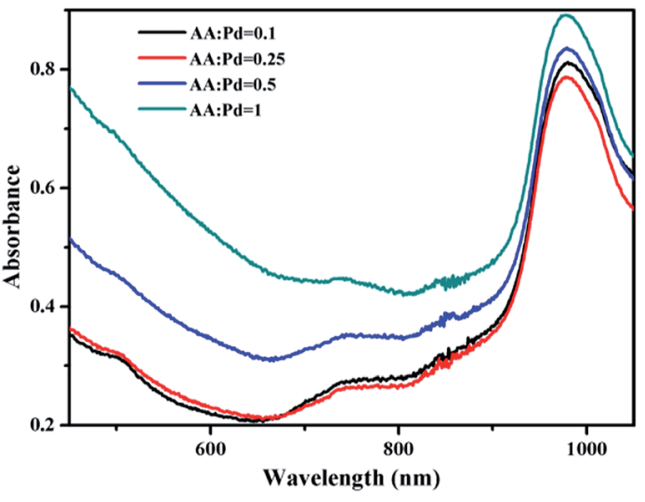

Fig. 4 UV-vis-NIR absorption spectra of the four samples.

can be seen in Fig. 6, the dissociated nanoshperes are also PdAg alloy, which is further supported that $\mathrm{Ag}^{+}$ions have great impact on forming polycrystalline Pd nanostructure. ${ }^{32}$

In order to compare the catalytic performance between heterostructure and homogeneous structure of Pd modified Au NRs, core-shell structure was further prepared. Fig. 7a shows Au@Pd core-shell nanocuboids. The as-prepared CTAB-capped Au NRs played as core, which was coated with a thin Pd layer with a thickness of $9 \mathrm{~nm}$. The homogeneously growth pattern of Pd layer belongs to Frank-van der Merwe mode, which was confirmed by HRTEM (Fig. 7b) that a single Pd layer was aligned along the same direction with Au NRs. ${ }^{334}$ The SEAD pattern in Fig. 7b indicates Au@Pd core-shell nanocuboids are single crystalline in nature with well defined face-centered cubic (fcc) structures.

Fig. 7c shows Au@Pd core-shell nanorods with irregular shapes. The CTAC-capped Au NRs were coated by a irregular Pd thin layer. Fig. 7d shows Pd layer presents a epitaxial growth pattern, while polycrystalline Pd particles were found growth on the surface of Pd thin layer. The SAED patterns (Fig. 7d) identify the Au NRs coated by Pd single crystalline with fcc structure. Some small polycrystalline Pd nanoparticles grow on the surface of Pd layer. This may be caused by CTAC soft template that is not able to specifically stabilize a particular crystalline facet like CTAB, leading to the irregularly shaped morphology. ${ }^{35}$
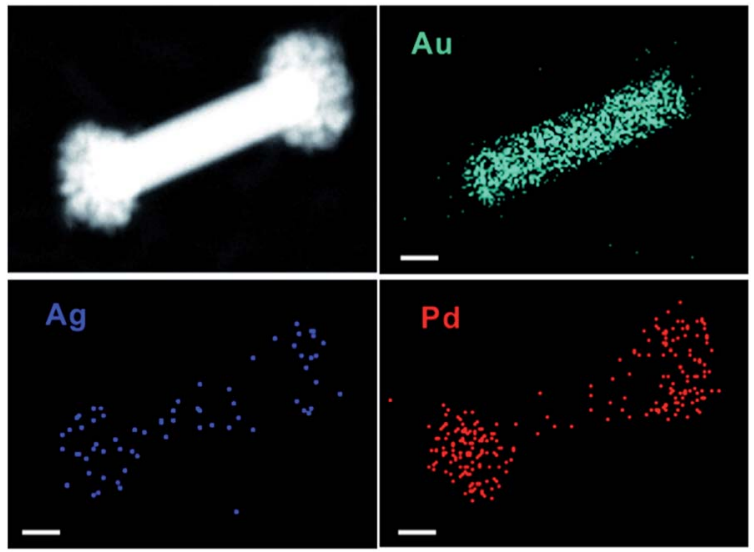

Fig. 5 STEM images and corresponding STEM-EDS elemental maps of $\mathrm{Au}, \mathrm{Pd}, \mathrm{Ag}$ of single Au/Pd NDBs, the scale bars represent $20 \mathrm{~nm}$.

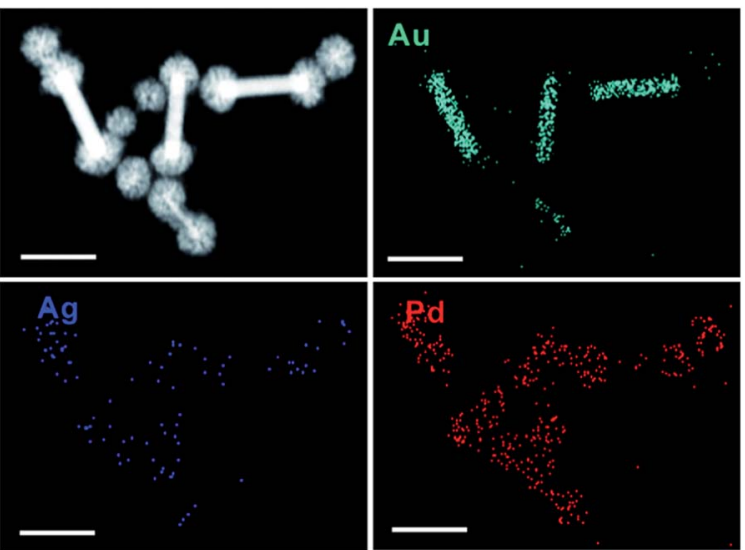

Fig. 6 STEM images and corresponding STEM-EDS elemental maps of Au/Pd NDBs and PdAg nanoshperes, the scale bars represent $100 \mathrm{~nm}$.

Fig. 7e shows Au@Pd core-shell nanorods with serrated shapes. The CTAB-capped Au NRs were coated with disconnected Pd thin layer in the Ag ions. HRTEM (Fig. 7f) image shows that the mass Pd nanoparticles with orientated growth along the (200) plane. SAED (Fig. 7f) pattern shows a single crystalline pattern of $\mathrm{Au}$ and $\mathrm{Pd}$. The formation of

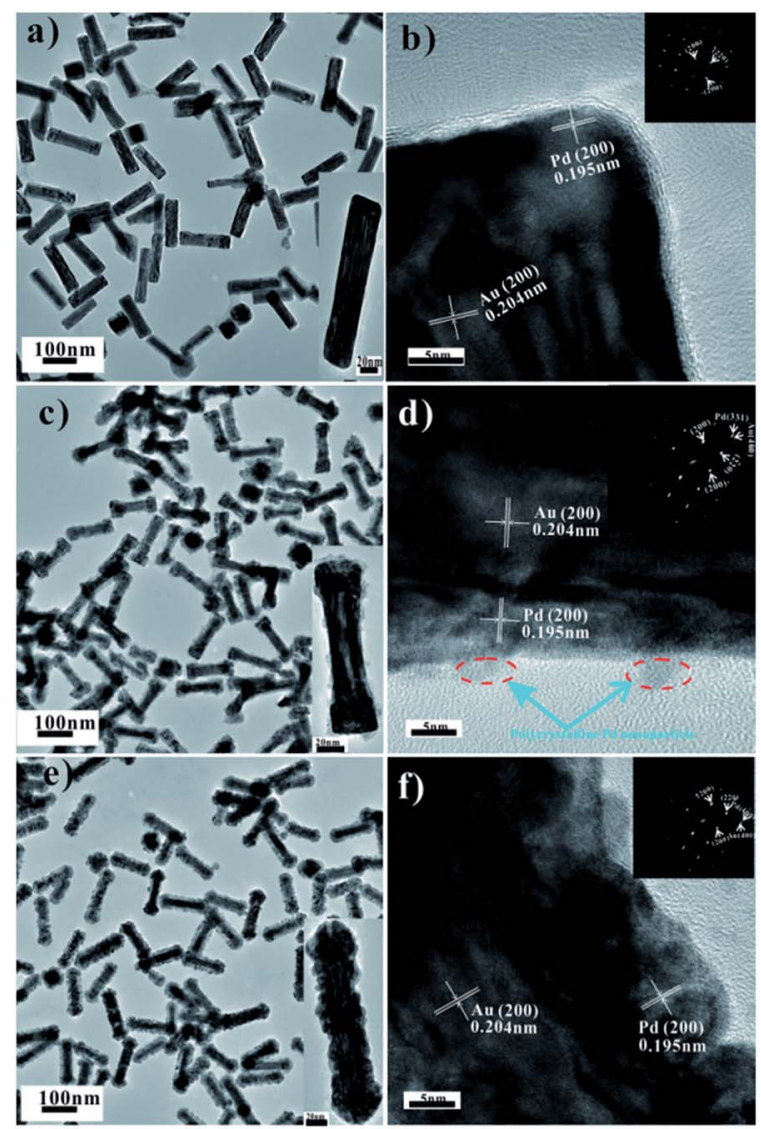

Fig. 7 TEM and HRTEM images: ( $a$ and b) AuaPd core-shell nanocuboids, (c and d) AuaPd core-shell nanostructure with irregular shapes, (e and f) Au@Pd core-shell nanorods with serrated shapes. 
discontinuous Pd shell due to the existence of the lattice vacancies generated by the galvanic replacement between $\mathrm{Ag}$ and $\mathrm{Pd}^{2+}$ in the modified procedure.

The most exciting feature of the designed Au/Pd NDBs offer the possibilities to promote SPR-induced hot-electron generation under visible and NIR light irradiation. The samples prepared in the presence of CTAB, CTAB- $\mathrm{Ag}^{+}$, CTAC and CTAC$\mathrm{Ag}^{+}$were treated with perchloric acid $\left(\mathrm{HClO}_{4}\right)$ to remove the coated surfactants (CTAB or CTAC) that hinder charge-transfer process before using in $\mathrm{H}_{2}$ production experiments. Fig. 8a shows different reaction rate of $\mathrm{H}_{2}$ evolution via formic acid dehydrogenation in the dark or under light irradiation at $15{ }^{\circ} \mathrm{C}$. TCD test only detected the presence of $\mathrm{H}_{2}$ and $\mathrm{CO}_{2}$, which conforms with the results of Pd tipped Au NRs catalysts for formic acid dehydrogenation. ${ }^{26}$ With light irradiation, the $\mathrm{H}_{2}$ evolution rate over $\mathrm{Au} / \mathrm{Pd}$ NDBs reached to $1.05 \mathrm{mmol} \mathrm{g}^{-1} \mathrm{~h}^{-1}$ while that in the dark do not shows catalytic performance. As can be seen from Fig. 8b, there is a difference in $\mathrm{H}_{2}$ production between $\mathrm{Pd}-\mathrm{Au}$ heterogeneous structure and $\mathrm{Pd}-\mathrm{Au}$ homogeneous structure. Au/Pd NDBs exhibited a high rate of $\mathrm{H}_{2}$ evolution while Au@Pd core-shell NP did not show catalytic ability. Fig. 8c and d shows the catalytic activity of Au/Pd NDBs under light irradiation. For $\mathrm{Au} / \mathrm{Pd} \mathrm{NDBs}, \mathrm{H}_{2}$ linearly evolved from the suspensions with increasing irradiation time. The $\mathrm{H}_{2}$ evolution rate initially arises with the increasing ball size. However, the rate drops to zero at $\mathrm{AA}: \mathrm{Pd}=1$.

$\mathrm{Au}$ NRs function as carrier and hot-electrons source for Pd nanoparticles. The dispersity of Pd particles and quantity of catalytic sites are two major factors that influence the $\mathrm{H}_{2}$ production in this experiment. Table 1 shows that the corresponding $\mathrm{H}_{2}$ evolution rate increases from $0.45 \mathrm{mmol} \mathrm{g}^{-1} \mathrm{~h}^{-1}$ to $1.05 \mathrm{mmol} \mathrm{g}^{-1} \mathrm{~h}^{-1}$ when the size of Pd particles increases from
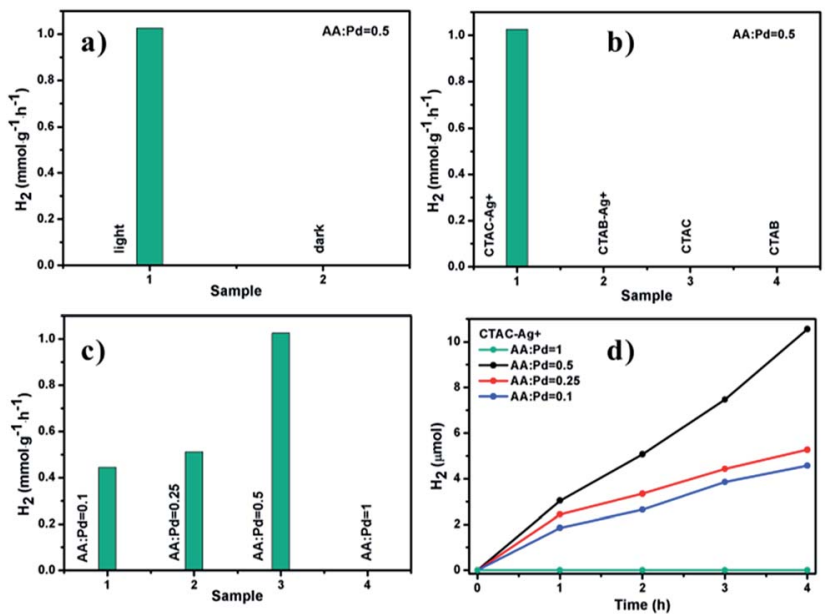

Fig. 8 (a) The rate of $\mathrm{H}_{2}$ evolution under dark/light irradiation over $\mathrm{Au} /$ Pd NDBs (AA : Pd = 0.5), (b) the rate of $\mathrm{H}_{2}$ evolution under the light irradiation over the catalysts obtained in the presence of CTAC- $\mathrm{Ag}^{+}$, $\mathrm{CTAB}-\mathrm{Ag}^{+}, \mathrm{CTAC}, \mathrm{CTAB},(\mathrm{c})$ effect of ball size of $\mathrm{Au} / \mathrm{Pd}$ NDBs on the rate $\mathrm{H}_{2}$ evolution under the light irradiation over the catalysts obtained at $A A$ : Pd ratio of $0.1,0.25,0.5$ and 1 , (d) the time-dependent profiles of $\mathrm{H}_{2}$ evolution over the $\mathrm{Au} / \mathrm{Pd}$ NDBs obtained at $\mathrm{AA}$ : Pd ratio of 0.1 , $0.25,0.5$ and 1. Light irradiation is $\lambda>420 \mathrm{~nm}, 100 \mathrm{~mW} \mathrm{~cm}^{-2}$ at $15^{\circ} \mathrm{C}$.
Table 1 Photocatalytic activity comparison of Au/Pd NDBs

\begin{tabular}{lll}
\hline Catalyst & $\begin{array}{l}\text { Size of Pd } \\
\text { nanoparticles }(\mathrm{nm})\end{array}$ & $\begin{array}{l}\text { Rate of } \mathrm{H}_{2} \text { evolution } \\
\left(\mathrm{mmol} \mathrm{g}^{-1} \mathrm{~h}^{-1}\right)\end{array}$ \\
\hline $\mathrm{Au} /$ Pd NDBs-0.10 & 13 & 0.45 \\
$\mathrm{Au} /$ Pd NDBs-0.25 & 24 & 0.51 \\
$\mathrm{Au} /$ Pd NDBs-0.50 & 30 & 1.05 \\
$\mathrm{Au} /$ Pd NDBs-1.00 & 44 & 0
\end{tabular}

$13 \mathrm{~nm}$ to $30 \mathrm{~nm}$. The enlarged Pd particles mean bigger surface area and more catalytic site for $\mathrm{H}_{2}$ production. TEM images in Fig. 3(a-c) show that Pd particles in the $\mathrm{Au} / \mathrm{Pd}$ NDBs samples have good dispersity. The improved photocatalytic performance was caused by the increased catalytic sites in Pd particles. However, Au/Pd NDBs-1.00 do not have any catalytic performance. TEM image in Fig. 3d shows that the ball of $\mathrm{Au} / \mathrm{Pd}$ NDBs-1.00 with large ball size are too dense, which impairs the dispersity of Pd particles and contributes to the worsen catalytic activity. Therefore, the optimal reaction rate can be obtained by tuning the ball size in a relatively high ratio of AA : $\mathrm{H}_{2} \mathrm{PdCl}_{4}$. The turnover frequency (TOF) over Au/Pd NDBs obtained at $\mathrm{AA}: \mathrm{Pd}=0.5$ without any additive was measured to be $2.1 \mathrm{~h}^{-1}$ at $15{ }^{\circ} \mathrm{C}$ under the light irradiation.

In order to examine function region of electric field amplification by SPR excitation and its potential contribution to the plasmon-induced enhancement of $\mathrm{H}_{2}$ production, the 3-D FDTD simulation were performed to present the spatial distribution of electric field intensity via varying incident polarization direction (Fig. 9a and b). Considering the random orientation of $\mathrm{Au} / \mathrm{Pd}$ NDBs in suspensions, two patterns of stimulate light polarization direction (parallel and perpendicular) were employed to the Au/Pd NDBs. The results shows that the SPR enhancement of electric field mainly focus on the balls, a)

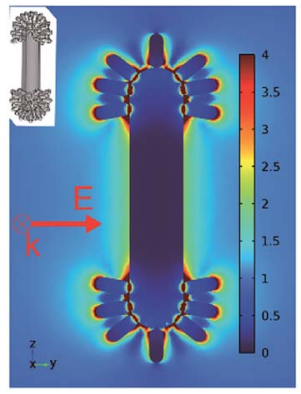

b)

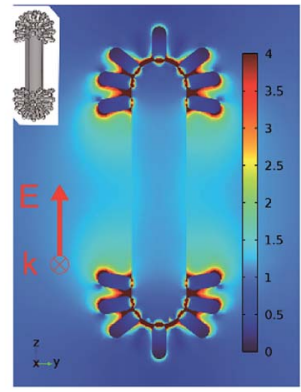

c)
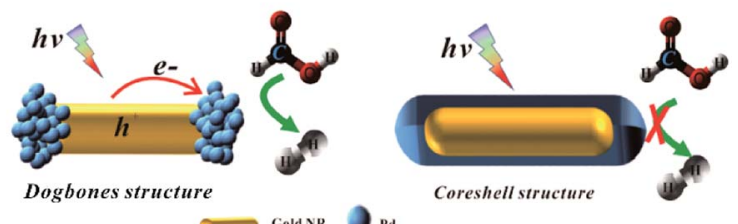

Fig. 9 ( $a$ and $b)$ Spatial distribution of the SPR-induced enhancement of electric field intensity for $\mathrm{Au} / \mathrm{Pd} \mathrm{NDBs}\left(\mathrm{AA}: \mathrm{H}_{2} \mathrm{PdCl}_{4}=0.5\right.$ ), simulated by FDTD method, here $E$ denotes the vector of the electric field and $k$ denotes the wavevector of $900 \mathrm{~nm}$ excitation laser, (c) reaction mechanism for $\mathrm{H}_{2}$ production. 
regardless of the polarization direction. This indicates that dogbones structure are effective for the occurrence of electron-hole pairs separation. Therefore, combining experimental results and FDTD simulation, a reasonable reaction mechanism for formic acid dehydrogenation over $\mathrm{Au} / \mathrm{Pd}$ NDBs under light irradiation is proposed, as illustrated in Fig. 9c. The NDBS structure are effective to harvest light to generate electron-hole pairs. Hot electrons accumulating in $\mathrm{Pd}$ surface improve the $\mathrm{H}_{2}$ catalytic performance. However, Fig. 9d shows the core-shell structure denied the opportunity for allowing longer-time electrons-hole separation due to the strongly charge recombination in homogeneous structure, leading to the absence of activity. ${ }^{36,37}$

\section{Conclusion}

In summary, an easy, bottom-up, wet-chemistry technique for the synthesis of $\mathrm{Au} / \mathrm{Pd}$ NDBs has been successfully developed by loading massive Pd nanoparticles on two ends of $\mathrm{Au}$ nanorods (NRs) via seed-mediated method. The Pd/Au NDBs shows higher enhanced reaction activity compared with samples with coreshell structure, which was attributed to the increased electron and hole pair separation and total catalytic size. More importantly, the introduction of $\mathrm{Au}$ NRs broadens the photoresponse range and improves the utilization of sunlight efficiently, which directs us to extend this work to other dogbones like heterostructure system, e.g. $\mathrm{Cu} / \mathrm{Pd} \mathrm{NDBs}$ and therefore provides new opportunities in designing advanced photoactive materials with high stability and enhanced performance for solar energy conversion.

\section{Conflict of interest}

There are no conflicts of interest to declare.

\section{Acknowledgements}

This study was supported by a fund from the National Natural Science Foundation of China (No. 51471182) and Shanghai International Science \& Technology Cooperation Program (No. 17520711700).

\section{Notes and references}

1 A. Kudo and Y. Miseki, Chem. Soc. Rev., 2009, 38, 253-278.

2 K. Maeda and K. Domen, J. Phys. Chem. Lett., 2010, 1, 2655-2661.

3 S. Linic, P. Christopher and D. B. Ingram, Nat. Mater., 2011, 10, 911-921.

4 C. Clavero, Nat. Photonics, 2014, 8, 95-103.

5 S. Mubeen, J. Lee, D. Liu, G. D. Stucky and M. Moskovits, Nano Lett., 2015, 15, 2132-2136.

6 X. Huang, Y. Li, Y. Chen, H. Zhou, X. Duan and Y. Huang, Angew. Chem., Int. Ed., 2013, 52, 6063-6067.

7 Z. Zheng, T. Tachikawa and T. Majima, J. Am. Chem. Soc., 2014, 136, 6870-6873.

8 B. Wu, D. Liu, S. Mubeen, T. T. Chuong, M. Moskovits and G. D. Stucky, J. Am. Chem. Soc., 2016, 138, 1114-1117.

9 M. Grasemann and G. Laurenczy, Energy Environ. Sci., 2012, 5, 8171-8181.
10 C. Hu, S. W. Ting and K. Y. Chan, Int. J. Hydrogen Energy, 2012, 37, 6372-6380.

11 S. Park, Y. Xie and M. J. Weaver, Langmuir, 2002, 18, 57925798.

12 K. Tedsree, T. Li, S. Jones, C. W. Chan, K. M. Yu, P. A. Bagot, E. A. Marquis, G. D. Smith and S. C. Tsang, Nat. Nanotechnol., 2011, 6, 302-307.

13 S. Fukuzumi, T. Kobayashi and T. Suenobu, J. Am. Chem. Soc., 2010, 132, 1496-1497.

14 X. Zhou, Y. Huang, W. Xing, C. Liu, J. Liao and T. Lu, Chem. Commun., 2008, 30, 3540-3542.

15 M. Ojeda and E. Iglesia, Angew. Chem., Int. Ed., 2009, 48, 4800-4803.

16 Z. L. Wang, J. M. Yan, Y. Ping, H. L. Wang, W. T. Zheng and Q. Jiang, Angew. Chem., Int. Ed., 2013, 52, 4406-4409.

17 Q. Y. Bi, X. L. Du, Y. M. Liu, Y. Cao, H. Y. He and K. N. Fan, J. Am. Chem. Soc., 2012, 134, 8926-8933.

18 Y. Cao, Y. Yang, Y. Shan and Z. Huang, ACS Appl. Mater. Interfaces, 2016, 8, 5998-6003.

19 N. V. Long, Y. Yang, C. M. Thi, N. V. Minh, Y. Cao and M. Nogami, Nano Energy, 2013, 2, 636-676.

20 A. Cuesta, G. Cabello, M. Osawa and C. Gutierrez, ACS Catal., 2012, 2, 728-738.

21 M. Ojeda and E. Iglesia, Angew. Chem., Int. Ed., 2009, 121, 4894-4897.

22 S. Jones, J. Qu, K. Tedsree, X. Q. Gong and S. C. E. Tsang, Angew. Chem., Int. Ed., 2012, 51, 11275.

23 Y. Huang, X. Zhou, M. Yin, C. Liu and W. Xing, Chem. Mater., 2010, 22, 5122-5128.

24 X. Gu, Z. Lu, H. Jiang, T. Akita and Q. Xu, J. Am. Chem. Soc., 2011, 133, 11822-11825.

25 S. Zhang, O. Metin, D. Su and S. Sun, Angew. Chem., Int. Ed., 2013, 52, 3681-3684.

26 Z. Zheng, T. Tachikawa and T. Majima, J. Am. Chem. Soc., 2015, 137, 948-957.

27 D. Y. Kim, K. W. Choi, X. L. Zhong, Z. Y. Li, S. H. Im and O. O. Park, CrystEngComm, 2013, 15, 3385-3391.

28 F. R. Fan, D. Y. Liu, Y. F. Wu, S. Duan, Z. X. Xie, Z. Y. Jiang and Z. Q. Tian, J. Am. Chem. Soc., 2008, 130, 6949-6950.

29 X. Ye, C. Zheng, J. Chen, Y. Gao and C. B. Murray, Nano Lett., 2013, 13, 765-771.

30 H. Jing and H. Wang, CrystEngComm, 2014, 16, 9469-9477.

31 P. B. Johnson and R. W. Christy, Phys. Rev. B: Solid State, 1972, 197(6), 4370-4379.

32 L. Zhang, J. Zhang, Z. Jiang, S. Xie, M. Jin, X. Han, Q. Kuang, Z. Xie and L. Zheng, J. Mater. Chem., 2011, 21, 9620-9625.

33 X. Xia, Y. Wang, A. Ruditskiy and Y. Xia, Adv. Mater., 2013, 25, 6313-6333.

34 H. Jing and H. Wang, CrystEngComm, 2014, 16, 9469-9477. 35 X. Guo, Q. Zhang, Y. H. Sun, Q. Zhao and J. Yang, ACS Nano, 2012, 6, 1165-1175.

36 S. K. Cushing, A. D. Bristow and N. Wu, Phys. Chem. Chem. Phys., 2015, 17, 30013-30022.

37 S. K. Cushing, J. Li, J. Bright, B. T. Yost, P. Zheng, A. D. Bristow and N. Wu, J. Phys. Chem. C, 2015, 119, 16239-16244. 